\title{
Benefits of Potato Peels
}

\author{
Nischala Susarla* \\ Dietician, Melbourne, Australia \\ *Corresponding Author: Nischala Susarla, Dietician, Melbourne, Australia.
}

Received: August 06, 2019; Published: August 20, 2019

DOI: 10.31080/ASNH.2019.03.0418

\begin{abstract}
The potato is a starchy, tuberous crop from the perennial nightshade Solanum tuberosum. The word "potato" may refer either to the plant itself or the edible tuber. Potato peels are considered as waste in many households as well as in the industries.

The peels contain an array of nutritionally beneficial compounds, which can be utilized in many ways, e.g. the peel extract, can be used as a natural antioxidant in foods. Moreover, the phenolic compunds are particularly beneficial in the treatment of certain chronic diseases, and also in the prevention of cancer. The potato's fiber, potassium, vitamin C and vitamin B-6 content, coupled with its lack of cholesterol, all support heart health. Varying amounts of potassium, iron, riboflavin, folate, and vitamins are found primarily in the thick periderm of the potato skin. The concentrations of some minerals were found to be greater in the skin than in the flesh of the tuber.
\end{abstract}

Keywords: Potato Peels; Flesh; Potato

\section{Introduction}

West Bengal ranks second in potato production after Uttar Pradesh, the leader in potato production, in the country. Both of them collectively contribute more than $50 \%$ of the country's production of potato. So any minor deviation in the production of these two states largely affects the total Indian production and market prices. Although India holds the $5^{\text {th }}$ place in cultivation after China, Russia, Poland and USA its share in export is very low. In world context, potato export from India does not stand mainly due to high prices and poor quality and inconsistency of produce. On the other hand, India has a dedicated potato research institute in the name of Central Potato Research Institute (CPRI) located in Shimla, Himachal Pradesh. HP is not one of the two major cultivation states.

Types of processed potato items available are

- $\quad$ Potato chips and wafers

- French Fries

- Potato Starch

- Potato Powder

- $\quad$ Flakes and Pellets

Potato is commonly believed to be unhealthy due to its high starch and high consumption when fried with oil. Consumption of potatoes in the right amount, right high bioactive variety and with low processing could potentially lead to the prevention of oxidation-linked chronic diseases such a type II diabetes cardiovascular diseases as opposed to its usual form of French fries and potato chips.

\section{Potato peels}

As a consequence of the processing of potato products in the industries, a lot of potato by-products are generated. One of them includes the potato peels.

Peels are the major by-product of potato processing industries, which represent a major waste disposal problem for the industry concerned. Up-grading of this by-product to value added products is therefore of interest to the potato industry.

The peeling is accomplished by abrasive peeling, steam peeling or lye peeling, depending on the types of products to be processed. Abrasive peeling is used for chips, whereas steam peeling is used for frozen and dehydrated products. The use of lye peeling requires a neutralization step, after peeling.

Potato skin is composed of suberized phellem cells, the outer component of the tuber periderm. The periderm tissue consists of two additional cell types: a single-cell meristematic layer, the phellogen (cork cambium) that produces the phellem cells and is localized underneath them; and a parenchyma-like phelloderm that is derived from inward cell divisions of the phellogen. The periderm is a tissue of secondary origin that replaces the epidermis when the latter is damaged.

Compounds present in potato peel

Potato peels contain an array of nutritionally and pharmacologically beneficial compounds. They are mainly

- $\quad$ Cell wall polysaccharides

- Dietary fibre 
- Antioxidants

- Phenolic compounds

- Vitamins and minerals

\section{Dietary fibre of potato peel}

- Approximately $50 \%$ of potato peels have dietary fibre.

- The primary constituent of potato fiber is a polysaccharide.

- $\quad$ Cellulose

- Hemicelluloses

- $\quad$ Liginis

Dietary fiber is well known as a bulking agent, increasing the intestinal mobility and hydration of the feces (13). Several authors have reviewed the importance of consumption of moderate amounts of dietary fibers for human health (14).

Scientifically speaking, dietary fiber is a broad term that includes several carbohydrates; cellulose, hemicelluloses, lignins, pectins, gums etc. Potato peel fibers are primarily insoluble, and can bind bile acids in-vitro. It is believed that binding of bile acids is one of the mechanisms whereby certain sources of dietary fibers lower plasma cholesterol. The hypocholesterolemic effect of dietary fiber from Potato Peel was studied and found that after four weeks of feeding on potato peels, rats showed $40 \%$ reduction in plasma cholesterol content and $30 \%$ of hepatic fat cholesterol levels were reduced as compared with animals fed only with cellulose supplemented diet. Defects of dietary fiber on lipid-profile influence several health related issues. High concentrations of low-density lipoprotein (LDL) cholesterol, other dyslipidemia (high concentration of triglycerides and low concentration of high-density lipoprotein [HDL] cholesterol), leads to blood platelets aggregation, risk factors for cardiovascular diseases (CVD), and hypertension. Moreover, high intake of dietary fibers has a positive influence on blood glucose profile and it is related health complications, in healthy and diabetic individuals of both types. By altering the gastric emptying time, dietary fibers are able to affect the absorption of other simple sugars. The effect of dietary fibers on blood glucose and insulin response has been demonstrated by many other authors as well.

Different sources of dietary fibers have been used to replace wheat flour in the preparation of bakery products. Potato peel (PP) was introduced as a promising source of dietary fiber. Since approximately $50 \%$ of potato peels $(\mathrm{w} / \mathrm{w})$ is dietary fibers. The physical and chemical characteristics of PP and reported PP as being superior to wheat bran in its content of total dietary fiber, water holding capacity, and low quantities of starchy components.

Wheat flour was also substituted with PP in the production of white bread, but it increased crumb darkening and reduced the loaf volume found that PP caused a musty odor in breads, but the extrusion of potato peel before its utilization can diminish this aroma from the final product. The effect of using PP in biscuit processing resulted in biscuits produced using 5 and $10 \%(\mathrm{w} / \mathrm{w})$ of PP replaced wheat flour were smaller in terms of their stack weight and sensory score (color and appearance) in proportion to the levels used. In addition, supplementary biscuits were harder to bite than control biscuits. It was also found by Camire., et al. 1994 that muffins with $25 \%$ PP were darker, lower in height and more resistant to compression. Similarly, cookies with 10 and 15\% PP $(\mathrm{w} / \mathrm{w})$ were darker, harder, and smaller in diameter than control cookies. From a different prospect it was suggested, the possible effect of potato peel as a good source of dietary fiber to be acting as anticarcinogenic material. Dietary fibers are known as protective materials against mutagenesis and carcinogenesis via several well established mechanisms that include binding of carcinogenic and mutagenic substances, reduce intestines-transit time, increase water absorption and fecal bulk, and lower fecal $\mathrm{pH}$ through the fermentation by intestinal microflora. The probable role of extruded vs. unextruded potato peel, in comparison with wheat bran and cellulose as other sources of dietary fiber, in binding of the carcinogenic benzopyrene in designed in-vitro digestion model were investigated. Un-extruded steam peels bound more benzopyrene than did peels extruded at temperature of $110^{\circ} \mathrm{C}$ and feed moisture of $30 \%$. Moreover, binding of benzopyrene by abrasion potato peels was lower than that by steam potato peels. In addition abrasion peels contain approximately $25 \%$ dietary fiber and $50 \%$ starch, which may have maintained benzopyrene in suspension and / or higher amount of polyphenols remaining in abrasion peel, in contrast with steam peel, which may also have role in lowering of levels of benzopyrene by interaction and binding. The method of peeling was found to be a key factor influencing the chemical composition of peels and its suitability for further utilization. Extrusion of potato peels was associated with an increase in total dietary fiber and lignin contents and a decrease in starch content in steam peels. Lignin content was found to decrease but total dietary fiber content was unaffected in extruded abrasion peels. Soluble nonstarch polysaccharides increased in both types of peeling because of extrusion. The higher quantity of glucose recovered from the insoluble fiber fraction of extruded steam peels was reported to be the possible reason in the formation of resistant starch. On the other hand, manual peeling of potato produced peels with approximately $63 \%$, on a dry weight basis of alcohol-insoluble fibers, which was separated into pectic substances, hemicellulose, cellulose, and lignin. These fractions consisted of $3.4 \%$ pectin, $2.2 \%$ cellulose, $14.7 \%$ protein, $66.8 \%$ starch, and $7.7 \%$ ash. The sugars in the alcohol-soluble fraction consisted of $1.4 \%$ total soluble sugars and $0.9 \%$ reducing sugars.

\section{Phenolic compounds in potato peel}

Phenolic compounds are heterogenous class of secondary metabolites which are classified as phenolic acids and flavonoids. The latter components represent a very large subclass, with approximately 9000 compunds. The major phenolic acids present were: gallic acid, caffeic acid, chlorogenic acid and protocatechuic acid. An experimental pro-oxidant system was used to induce lipid peroxidation in rat red blood cells (RBCs) and human RBC membranes. PP was found to inhibit lipid peroxidation with similar effectiveness in both the systems (about $80-85 \%$ inhibition by PP at 
$2.5 \mathrm{mg} / \mathrm{ml}$ ). The following are the phenolic compounds present in the skin of potato.

Phenolic compounds are the main class of natural antioxiadants. extracts of peels from Sava variety of potatoes were highly efficient in reducing lipid peroxidation both in fish oil and in oil-inwater emulsions (Habeebullah., et al. 2010).

- Chlorogenic (50.31\%),

- $\quad$ Gallic (41.67\%)

- $\quad$ Protocatechuic (7.815\%)

- $\quad$ Caffeic acids(0.21\%)

- $\quad$ Ferulic acid

- $\quad$ Vanillic acid

- $\quad$ Salicyclic acids

The flavonoids in the potato skin are

- Quercetin

- Naringenin

- Catechin

- $\quad$ Epicatechin

Chlorogenic acid is by far the most abundant phenolic component and constitutes upto $90 \%$ of the total phenolics. It has a Protective effect in neuroinflammatory conditions and the ability to lower blood pressure in hypertensive patients, and its derivative has shown to lower blood pressure in hypertensive rats (Cheplick, 2010).

- Caffeic acid is an antioxidant in vitro and also in vivo. Caffeic acid also shows immunomodulatory and antiinflammatory activity (J.H. Park., et al. 2004). Caffeic acid outperformed the other antioxidants, reducing aflatoxin production by more than 95 percent. The studies are the first to show that oxidative stress that would otherwise trigger or enhance Aspergillus flavus aflatoxin production can be stymied by caffeic acid. This opens the door to using natural fungicide methods by supplementing trees with antioxidants.

- Antibacterial activities of these compounds were found in high doses against Escherechia coli, Salmonella typhimurium and Bacillus cereus.

- Antioxidant activity was found in multiple systems such as superoxide scavenging ability.

- Quercetin, a flavonoid found in potato skin, possesses powerful anti-inflammatory properties and antioxidant capabilities that protect the body's cells from free radical damage.

- The hydroxycinnamic acids of potato peel also caused in vitro peroxidation of human low density lipoprotein (LDL).
- Catechin activity in potato indicates the prevention of type II diabetes (Matsui., et al. 2007), cardiovascular complications and cancer prevention abilities (Nagao., et al. 2007).

- The compounds showed antioxidative activities similar to BHA and BHT in inhibiting lipid oxidation in sunflower oil.

- Choline is a very important and versatile nutrient in potatoes that helps with sleep, muscle movement, learning and memory. Choline also helps to maintain the structure of cellular membranes, aids in the transmission of nerve impulses, assists in the absorption of fat and reduces chronic inflammation.

- The protein contained in potato skins which is known as patatin, can help in reducing blood pressure and shield the heart against possible disease.

- In a study done by Lorenzo., et al, 2001; the extracts of potato peels blocked the migration of tumor cells in vitro. (Sarcomatoid carcinoma), in mice.

\section{Nutritional composition of raw potato with skin}

Varying amounts of potassium, iron, riboflavin, folate, and vitamins are found primarily in the thick periderm of the potato skin. The concentrations of some minerals were found to be greater in the skin than in the flesh of the tuber. The table indicates the nutritional value of raw potatoes with the skin.

\begin{tabular}{|l|c|}
\hline \multicolumn{2}{|c|}{ Potato, raw, with skin } \\
\hline \multicolumn{2}{|c|}{ Nutritional value per 100 g (3.5 oz) } \\
\hline Energy & $321 \mathrm{~kJ}(77 \mathrm{kcal})$ \\
\hline Carbohydrates & $17.47 \mathrm{~g}$ \\
\hline Starch & $15.44 \mathrm{~g}$ \\
\hline Dietary fiber & $2.2 \mathrm{~g}$ \\
\hline Fat & $0.1 \mathrm{~g}$ \\
\hline Protein & $2 \mathrm{~g}$ \\
\hline \multicolumn{2}{|c|}{ Vitamins } \\
\hline Thiamine (B1) & $(7 \%)$ \\
\hline Riboflavin (B2) & $0.08 \mathrm{mg}$ \\
\hline Niacin (B3) & $(3 \%)$ \\
\hline Pantothenic & $0.03 \mathrm{mg}$ \\
acid (B5) & $(7 \%)$ \\
\hline \multirow{2}{*}{ Vitamin B6 } & $1.05 \mathrm{mg}$ \\
\hline Folate (B9) & $(6 \%)$ \\
\hline Vitamin C & $0.296 \mathrm{mg}$ \\
\hline
\end{tabular}




\begin{tabular}{|l|c|}
\hline Vitamin E & $(0 \%)$ \\
& $0.01 \mathrm{mg}$ \\
\hline Vitamin K & $(2 \%)$ \\
& $1.9 \mu \mathrm{g}$ \\
\hline \multicolumn{2}{|c|}{ Trace metals } \\
\hline Calcium & $(1 \%)$ \\
& $12 \mathrm{mg}$ \\
& \\
\hline Iron & $(6 \%)$ \\
& $0.78 \mathrm{mg}$ \\
\hline Magnesium & $(6 \%)$ \\
& $23 \mathrm{mg}$ \\
\hline Manganese & $(7 \%)$ \\
& $0.153 \mathrm{mg}$ \\
\hline Phosphorus & $(8 \%)$ \\
& $57 \mathrm{mg}$ \\
\hline Potassium & $(9 \%)$ \\
& $421 \mathrm{mg}$ \\
\hline Sodium & $(0 \%)$ \\
& $6 \mathrm{mg}$ \\
\hline Zinc & $(3 \%)$ \\
& $0.29 \mathrm{mg}$ \\
\hline
\end{tabular}

Table

Source: USDA Nutrient database.

- Collagen, the skin's support system, relies on vitamin C as an essential nutrient that works in our bodies as an antioxidant to help prevent damage caused by the sun, pollution and smoke. Vitamin C also promotes collagen's ability to smooth wrinkles and improve overall skin texture.

- The potato's fiber, potassium, vitamin C and vitamin B-6 content, coupled with its lack of cholesterol, all support heart health.

- Potatoes contain significant amounts of fiber, which helps lower the total amount of cholesterol in the blood, thereby decreasing the risk of heart disease. In one study, those who consumed $4069 \mathrm{mg}$ of potassium per day had a 49\% lower risk of death from ischemic heart disease compared to those who consumed less potassium (about $1000 \mathrm{mg}$ per day).

- Vitamin B-6 prevents the buildup of a compound known as homocysteine. When excessive amounts of homocysteine accumulate in the body, it can damage blood vessels and lead to heart problems.

Glycoalkaloids in potato peels

The principle glycoalkaloids in potatoes are a-solanine and achaconine. They contribute flavour to potatoes but at higher concentrations cause bitterness and are toxic to humans. Their natural function is probably to serve as stress metabolites or phytoalexins for the protection of the potato when attacked by insects, fungi, etc. Increases of solanine in the potato peel are closely associated with greening (synthesis of chlorophyll) of the peel. These biochemical processes are independent of each other but are both activated by light.

For food safety purposes, an upper limit for glycoalkaloid content of $20 \mathrm{mg}$ per $100 \mathrm{~g}$ of potato is generally accepted $(100 \mathrm{~g}$ is slightly less than $1 / 4 \mathrm{lb}$ ). Concentrations of glycoalkaloids are 3 to 10 times greater in the peel than in the flesh. The average content in the peel can vary from 3 to more than $100 \mathrm{mg} / 100 \mathrm{~g}$ of peel. For peeled potatoes, the average content can vary from 0.10 to 4.50 $\mathrm{mg} / 100 \mathrm{~g}$

The potato alkaloids exert their toxic effects on the nervous system by interfering with the body's ability to regulate acetylcholine, a chemical responsible for conducting nerve impulses. Potato glycoalkaloids also act by general disruption of membranes, and symptoms of solanine toxicity include headache, nausea, fatigue, vomiting, abdominal pain and diarrhea. Cooking potatoes does not destroy the solanine. The glycoalkaloid a-chaconine is considered more toxic than a-solanine. Temporary gastrointestinal problems have been reported for some individuals eating potatoes that contained 3-10 mg/100g glycoalkaloids.

However, the rate of toxicity depends on post harvest conditions, such as storage, exposure to light, mechanical injury, etc The green colouration of the peels and the flesh is the indication of toxicity. In a study done by Vronen., et al, in 2004, potato glycoalkaloids were used in the synthesis of steroid hormones, specially 16-dehydropregnenolone acetate (DPA).

Certain coloured varieties of potatoes, namely Mountain rose, Purple Majesty and Rio Grande, are rich in anthocyanins. They contain carotenoids, xanthophylls, lutein, etc. These potatoes are rare and are available only in certain parts of Europe.

\section{Tips for selection of potatoes}

- Select potatoes that are firm, un-bruised and relatively smooth and round.

- Be sure that the potatoes are not sprouting or have green discoloration, as these are indicators of the presence of the toxic compound solanine.

- $\quad$ Potatoes should be stored between 45-50 degrees $\mathrm{F}$ in a dark, dry environment

\section{Miscellaneous uses of potato peels}

- Potato peel used as a low-cost agroindustrial medium in production of both alpha-amylase and alkaline protease enzymes and several extracellular hydrolytic enzymes which produced high yield and high activity of the produced enzymes. Also, an industrially important polysaccharide 'pullulan', can be produced by enzymatic hydrolysis of potato processing wastes. 
- It was extensively used in different food industries, under solid-state controlled growth conditions and was successfully used in some applications.

- Potato peel is used in livestock and cattle feed, since the fibres are suitable for ruminants, and it also contains certain nutrients beneficial for the animal.

- The potato peels may be used as a replacer of wood fibre in paper making. This is a very cheap and efficient method of utilization.

- The potato processing wastes are used as a substrate for yeast production and Vitamin B12 production.

\section{Studies}

Tamsulosin-induced hepatotoxicity and nephrotoxicity and its prevention by potato peel extract

S. al-jassabi., et al. American-Eurasian journal of toxicological sciences, 3(2): 52-58,2011

Aim: The aim of the study was to investigate the toxicity of an anticancer drug Tamsulosin by measuring its ability to induce ROS formation in the liver and kindney and the role of potato peel extract in the prevention of this damage.

Materials and Methods: The total phenolics was extracted from fresh potato peels. 60 mice were used in the study assigned into four groups (having 15 mice each). First group was the control which did not receive TAM(Tamsulosin) and PPE supplements. Group 2 received PPE $(100 \mathrm{mg} / \mathrm{Kg} /$ day), Group 3 received only TAM (400 microgm /kg daily), group 4 received both TAM and PPE (Potato Peel extract)

Results: The liver and kidneys of the mice were analyzed. It was observed that TAM treated mice had intrahepatic hemorrhage. PEE treated mice had a significantly lower levels of ROS formation. In Group 4 where both TAM and PPE were given, PPE was able to inhibit the toxicity of TAM in the kidney and liver.

Conclusion: Tamsulosin is a drug consumed all over the world, and potato peel extract comprises reasonable levels of polyphenols to act against toxicity of Tamsulosin.

Potato peel extract as a natural antioxidant in chilled storage of minced horse mackerel (Trachurus trachurus): Effect on lipid and protein oxidation K.H. Sabeena Farvin, Helene Drejer Grejsen, Charlotte Jacobsen, Food Chemistry 131 (2012) 843851

Aim: The present work was undertaken to examine the utilisation of potato peel, a waste material, as a source of natural antioxidants for retarding lipid and protein oxidation in minced mackerel.

Materials and Methods: The potato peels were dried in a hot air oven at $45 \mathrm{C}$ for $72 \mathrm{~h}$ and powdered. For the preparation of ethanol extract, $5 \mathrm{~g}$ of powdered peel was extracted with $50 \mathrm{ml}$ of $96 \%$ ethanol. The fish were beheaded and eviscerated immediately after catch. $600 \mathrm{~g}$ each of fillets was minced. The fillets was divided as Control, where 2.4 water extract (WE), 4.8 WE, 2.4,EE Ethanol Extracts of potato peel and 4.8 EE was added.

Results and Discussion: The addition of the ethanol extracts of potato peel at 2.4 and $4.8 \mathrm{~g} / \mathrm{kg}$ provided best protection towards lipid and protein oxidation in minced horse mackerel. The high efficacy of the ethanol extracts correlated with higher total phenolics and caffeic acid content in these extracts. The ethanol extracts were also shown to reduce tocopherol loss, indicating a tocopherol sparing property of the phenolic compounds. The water extracts showed lower efficacy and/or pro-oxidative effects especially when added in high concentration.

Conclusion: Thus, ethanol extracts of potato peel can be employed as natural antioxidant to prevent lipid and protein oxidation of fish fillets/mince in chilled storage of mackerel.

A comparative evaluation of antioxidant and antidiabetic potential of peel from young and matured potato

K.B. Arun, Janu Chandran, Food BioscienceVolume 9, 2015, Pages 36-46

Aim: In the present study antioxidant and antidiabetic potentials of potato peels at two different stages of maturity were evaluated and compared.

Materials and Methods: Peels of young and mature potatoes (YP and MP) were sequentially extracted with hexane (HMP, HYP), ethyl acetate (EMP, EYP) and methanol (MMP, MYP). EMP and EYP were found to possess the highest phenolic content (83.2 and 44.14 mg GAE/g dry weight, respectively) and maximum radical scavenging efficacy for different antioxidant assays performed

Results and Discussion: EYP demonstrated better $\alpha$-glucosidase inhibition activity (IC50-197.13 $\mu \mathrm{g}$ ), intracellular ROS scavenging and induce glucose uptake in L6 rat skeletal muscle cells. Phenolic profiling of compounds (gallic, caffeic, ferulic and chlorogenic acids) in the active extracts were established using HPLC. The study demonstrated that YP exhibited better bioactive potential than that of MP. YP could be an excellent source of bioactive phytochemicals with antioxidant and antidiabetic potential.

Conclusion: Young and matured potatoes could be an excellent source of bioactive phytochemicals with antioxidant and antidiabetic potential.

Effect of peeling and three cooking methods on the content of selected phytochemicals in potato tubers with various colour of flesh

Jaromír Lachman, Karel Hamouz, Food Chemistry Volume 138, Issues 2-3, 1 June 2013, Pages 1189-1197

Aim: The impact of peeling and three cooking treatments (boiling, baking and microwaving) on the content of selected phytochemicals in white-, yellow-, red- and purple-fleshed potatoes was investigated. 
Materials and Methods: Ascorbic acid and chlorogenic acid contents were determined by HPLC-DAD, total anthocyanin content by pH-differential spectrophotometry, glycoalkaloid, $\alpha$-chaconine and $\alpha$-solanine contents by HPLC-ESI/MS/MS. All cooking treatments reduced ascorbic and chlorogenic acid contents, total glycoalkaloids, $\alpha$-chaconine and $\alpha$-solanine with the exception of total anthocyanins.

Results and Discussion: The losses of ascorbic and chlorogenic acids were minimised with boiling and total anthocyanin levels retained the highest. Boiling of peeled tubers decreased contents of total glycoalkaloids ( $\alpha$-chaconine and $\alpha$-solanine) and appeared as the most favourable among the three tested methods. Moreover, due to higher initial levels, red- and purple-fleshed cultivars retained higher amounts of antioxidants (ascorbic acid, chlorogenic acid and total anthocyanin) after boiling and may be healthier as compared with white or yellow cultivars.

Conclusion: The red and purple fleshed cultivars retained higher amounts of anthocyanins and antioxidants than white or yellow cultivars.

Protective Effect of Potato Peel Powder in Ameliorating Oxidative Stress in Streptozotocin Diabetic Rats

Nandita Singh., et al. Plant Foods for Human NutritionJune 2005, Volume 60, Issue 2 pp 49-54

Aim: The potential of dietary potato peel (PP) powder in ameliorating oxidative stress (OS) and hyperglycemia was investigated in streptozotocin (STZ)-induced diabetic rats.

Materials and Methods: In a 4-week feeding trial, incorporation of potato peel powder ( 5 and $10 \%$ ) in the diet of diabetic rats was found to significantly reduce the plasma glucose level and also reduce drastically the polyuria of STZ diabetic rats.

Results and Discussion: The total food intake was significantly reduced in the diabetic rats fed $10 \%$ PP powder compared to the control diabetic rats. However, the body weight gain over 28 days was nearly four times greater in PP powder supplemented diabetic rats (both at 5 and 10\%) compared to the control diabetic rats. PP powder in the diet also decreased the elevated activities of serum transaminases (ALT and AST) and nearly normalized the hepatic MDA and GSH levels as well as the activities of specific antioxidant enzymes in liver of diabetic rats. The result of these studies clearly establishes the modulatory propensity of PP against diabetes induced alterations. Considering that potato peels are discarded as waste and not effectively utilized, these results suggest the possibility that PP waste could be effectively used as an ingredient in health and functional food to ameliorate certain disease states such as diabetes.

Conclusion: In a 4-week feeding trial, incorporation of potato pee powder ( 5 and $10 \%$ ) in the diet of diabetic rats was found to significantly reduce the plasma glucose level and also reduce drastically the polyuria of STZ diabetic rats.

\section{Conclusion}

Potato peels are considered as waste in many households as well as in the industries.

The peels contain an array of nutritionally beneficial compounds, which can be utilized in many ways, e.g. the peel extract, can be used as a natural antioxidant in foods. Moreover, the phenolic compunds are particularly beneficial in the treatment of certain chronic diseases, and also in the prevention of cancer.

\section{Bibliography}

1. Casas Lourdes., et al. "High Pressure Extraction of Antioxi dants from Solanum stenotomun Peel". Molecules 18.3 (2013): 3137-3151.

2. Azadeh Samarin. "Phenolics in Potato Peels: Extraction and Utilization as Natural Antioxidants". World Applied Sciences Journal 18.2 (2012): 191-195.

3. ScheieberAndreas., et al. "Potato Peels: A source of nutritionally and pharmacologically Interesting compounds- A Review". Global Science Books 2 (2008): 23-29.

4. Al-Weshahy. "Potato Peel as a Source of Important Phytochemical Antioxidant Nutraceuticals and Their Role in Human Health - A Review". Phytochemicals as Nutraceuticals - Global Approaches to Their Role in Nutrition and Health, Dr Venketeshwer Rao (Ed.), InTech (2012).

5. Kazuhiro Nara., et al. "Antioxidative activity of Bound-form phenolics in potato peel". Bioscience Biotechnology and Biochemistry 70.6 (2006): 1489-1491.

6. Parashar Sonia., et al. "Antimicrobial and antioxidant activities of fruits and vegetables- A review". Jounal of Pharmacognosy and Phytochemistry 3.1 (2014): 160-164.

7. Sal Jassabi., et al. "Tamsulosin induced Hepatotoxicity and its prevention by potato peel extract". American -Eurasian Journal of Toxicological Sciences 3.2 (2011): 52-58.

8. Jacobsen Charlotte. "Potato peel extract as a natural antioxidant in chilled storage of minced horse mackerel (Trachurus trachurus): Effect on lipid and protein oxidation". Food Chemistry 131.1 (2012): 843-851.

9. KB Arun., et al. "A comparative evaluation of antioxidant and antidiabetic potential of peel from young and matured potato". Food Bioscience 9.3 (2015): 36-46.

10. Lachman Jaromír. "Effect of peeling and three cooking methods on the content of selected phytochemicals in potato tubers with various colour of flesh". Food Chemistry 138.2-3 (2013): 1189-1197. 
11. Singh Nandita., et al. "Protective Effect of Potato Peel Powder in Ameliorating Oxidative Stress in Streptozotocin Diabetic Rats". Plant Foods for Human Nutrition 60.2 (2005): 49-54.

12. Vronen Patrick., et al. "The synthesis of 16-dehydropregnenolone acetate (DPA) from potato glycoalkaloids". ARKIVOC 14.24 (2004): 24-50.

Volume 3 Issue 9 September 2019

(C) All rights are reserved by Nischala Susarla. 